\title{
Role of Cardiac Biomarkers in the Assessment of Acute Cerebrovascular Accident
}

\author{
Srinivasan Radhakrishnan ${ }^{1}$ Swathy Moorthy ${ }^{1} \quad$ Sudish Gadde ${ }^{1} \quad$ Krishnaswamy Madhavan $^{1}$ \\ ${ }^{1}$ Department of General Medicine, Sri Ramachandra Medical \\ College, SRIHER, Chennai, Tamil Nadu, India

\begin{abstract}
Address for correspondence Swathy Moorthy, Department of General Medicine, Sri Ramachandra Medical College, SRIHER, Chennai, Tamil Nadu, 600116, India

(e-mail: drswathymoorthy@sriramachandra.edu.in).
\end{abstract}

J Neurosci Rural Pract:2021;12:106-111

\begin{abstract}
Background Stroke (cerebrovascular accident) has for long been a global burden in terms of its morbidity and mortality. Serum levels of cardiac enzymes such as creatine kinase-MB (CK-MB) component, troponin $\mathrm{T}$, and brain natriuretic peptide have been found to be elevated among the patients with stroke and also serve to prognosticate these patients. The serum levels of these enzymes correlate directly to the severity of stroke in these patients.

Objective Elevated cardiac enzymes among patients with acute cerebrovascular accidents are not uncommon despite the patients not having any cardiac problems. We aimed to identify the occurrence of elevated cardiac enzymes among patients with acute stroke and their correlation with the severity of stroke.

Materials and Methods Our study included 100 patients of acute stroke with no previous history of cardiac ailments. Serum levels of troponin I and CK-MB were analyzed among these patients using enzyme-linked immunosorbent assay method within the first 2 hours of admission. Patients outcome during the hospital stay were analyzed. Stroke severity was assessed using the National Institute of Health Stroke score (NIHS score) and the modified Rankin Score (mRS). The cardiac enzyme levels were correlated with these scores.

Results Twenty-eight percent of patients had elevated troponin I, while $72 \%$ patients had normal levels with the mean values of 10.36 to $106.54 \mathrm{ng} / \mathrm{mL}$ and 0.00 to $0.02 \mathrm{ng} /$ $\mathrm{mL}$, respectively. CK-MB levels were found elevated among $14 \%$ patients and normal among $86 \%$ patients with mean values of 5.8 to 124.36 and 0.0 to $4.3 \mathrm{ng} / \mathrm{mL}$, respectively. Among the six patients who succumbed to death, three patients had increased troponin I and four had elevated CK-MB. NIHS scores of $21.0357 \pm 6.79$ and $105.277 \pm 5.564$ were seen in patients with elevated and normal troponin I, whereas

Keywords

- stroke

- creatine kinase

- CK-MB

- troponin I

- cardiac enzymes

- cerebrovascular accident

NIHS scores of $20.4285 \pm 8.658$ and $11.8721 \pm 9.273$ were seen among patients with increased and normal CK-MB, respectively. The mRS scores were 4.3214 \pm 0.367 , $2.4305 \pm 1.374,4.2143 \pm 1.412$, and $2.756 \pm 1.749 \mathrm{ng} / \mathrm{mL}$ among the patients with elevated and normal troponin I and CK-MB, respectively.

Conclusion The mean values of cardiac enzymes troponin I and CK-MB were higher among patients with higher scores of NIHS and mRS. Among them, troponin I was very significant and it may serve as an early biomarker for the severity of stroke and hint on early cardiac evaluation among these patients.
\end{abstract}

DOI https://doi.org/

10.1055/s-0040-1721198 ISSN 0976-3147.
(C) 2021. Association for Helping Neurosurgical Sick People.

This is an open access article published by Thieme under the terms of the Creative Commons Attribution-NonDerivative-NonCommercial-License, permitting copying and reproduction so long as the original work is given appropriate credit. Contents may not be used for commercial purposes, or adapted, remixed, transformed or built upon. (https://creativecommons.org/licenses/by-nc-nd/4.0/)

Thieme Medical and Scientific Publishers Pvt. Ltd. A-12, 2nd Floor, Sector 2, Noida-201301 UP, India 


\section{Introduction}

Cerebrovascular accident also called stroke can be defined as a syndrome of abrupt onset of focal neurological deficits due to altered vascularity, causing ischemia or hemorrhage, resulting in complete or incomplete loss of brain functions. The devastating consequences of stroke make it a concerning issue. ${ }^{1}$ The World Health Organization foresees stroke as an imminent reason of disability and mortality in $2020 .{ }^{2}$ Early identification of stroke-related morbidity and mortality may help to reduce the mortality and improve the functional outcome by improvising newer and prompt management strategies in the at-risk population. ${ }^{3}$ Alterations in the central nervous system metabolism have long been known to influence the cardiac functions. ${ }^{4}$ Cardiac changes that happen in the stroke patients are firmly related to the excessive sympathetic nervous system activation that occurs as a result of damage to the insular cortex. ${ }^{5}$ Cardiac biomarkers such as troponin $\mathrm{T}$ and creatine kinase-MB (CK-MB) have also shown to increase in some patients with acute cerebrovascular accident without any cardiac injury. ${ }^{6}$

In an acute cerebrovascular accident, the presence of elevated cardiac enzymes could be either a secondary cardioembolic complication in a patient with primary myocardial injury or the myocardial injury could be a secondary insult in a patient with primary cerebral ischemia. Central activation of the sympathoadrenal system is known to occur in some cases of acute cerebrovascular accidents that could result in cardiac sequalae such as cardiac myocytolysis, elevation of cardiac enzymes, and arrhythmogenic disturbances. ${ }^{7}$ The brain has the CK-BB subunit; this can also explain the possible brain damage-induced secondary rise of CK-MB. ${ }^{8}$ Our study was aimed at estimating biomarkers namely troponin I and CK-MB among victims of acute cerebrovascular accidents without prior cardiac ailments.

\section{Materials and Methods}

Conducted in a South Indian hospital with referral standards, the project spanned duration of 18 months from April 2017 to September 2018. We included 263 patients admitted with acute cerebrovascular accidents among whom 161 patients were excluded due to either previous history of cardiovascular disease, electrolyte abnormalities, or previously documented abnormalities in the electrocardiograms (ECG) of these patients, while two patients did not consent to be involved and 100 of them were studied further. The commencement of the study was preceded by due approval by the ethics committee. A detailed history of the complaints, general and systemic examination were recorded in a prestructured proforma along with the basic blood investigations, ECG changes, cardiac biomarkers, and the type of stroke in neuroimaging. National Institute of Health Stroke Score (NIHS) and the modified Rankin Score (mRS) were used to assess stroke severity (the abbreviations cannot be modified). The state of stroke sufferers was noted at the end of their hospital stay. The correlation between the scores calculated and the cardiac enzymes was further analyzed.

\section{Statistics}

SPSS Software version 21 was employed to analyze the variables. Categorical variables were given as frequencies; the same were analyzed by chi-squared or Fischer's exact test. The tests were two sided and a $p$-value less than 0.05 was considered statistically significant.

\section{Results}

The study had 100 patients with stroke. Ischemic stroke was more common among our study population (78\%) than the hemorrhagic stroke. Twelve percent of our patients were less than 40 years of age and the incidence of ischemic stroke among them was significantly higher ( $p$-value 0.007). Males were two times more commonly involved than the females (2.03:1). Type 2 diabetes mellitus was the commonest comorbid condition ( $p$-value 0.034 ) compared with systemic hypertension and dyslipidemia. Similarly smoking had a strong association with the stroke occurrence ( $p$-value 0.004) compared with alcohol consumption. Elevated systolic and diastolic blood pressure were significantly associated with hemorrhagic stroke ( $p$-value $<0.001$ ).

Troponin I levels were found to be elevated among $28 \%$ of our study group compared with $14 \%$ patients having elevated CK-MB. The mean values of troponin I levels were 10.36 to $106.54 \mathrm{ng} / \mathrm{mL}$ and 0.00 to $0.02 \mathrm{ng} / \mathrm{mL}$ among the elevated and normal groups, whereas CK-MB levels were 5.8 to $96.73 \mathrm{ng} / \mathrm{mL}$ and 0.0 to $4.3 \mathrm{ng} / \mathrm{mL}$ among the elevated and normal groups, respectively.

The correlation of the common clinical parameters with normal or elevated troponin I and CK-MB is presented in - Table 1. Higher age groups were significantly associated with elevated cardiac enzymes. Similarly, we observed that the high values of troponin I and CK-MB had a strong association with worrisome NIHS and mRS scores at admission, prolonged hospital stay, and evidence of regional wall motion abnormality on two-dimensional (2D) echocardiogram. However, increased troponin I levels were seen among patients with higher diastolic blood pressures.

- Table 2 compares the NIHS scores among individuals with elevated and acceptable levels of troponin I and CK-MB. There was a strong correlation between higher NIHS scores with elevated cardiac enzymes. - Table 3 correlates the mRS scores to the elevated and normal levels of troponin I and creatine phosphokinase-MB (CPK-MB) among the stroke patients. Higher $\mathrm{mRs}$ scores were associated with elevated levels of cardiac enzymes.

- Table 4 shows the mean NIHS and mRS scores among the patients with elevated and normal levels of troponin $I$ and CK-MB that were statistically significant ( $p$-value $<0.001$ ). - Table 5 presents the comparison of parameters among patients with elevated troponin I and CK-MB levels that highlights troponin I elevation as a more significant cardiac enzyme to be associated with patients with acute stroke. Left ventricular hypertrophy (LVH) was strongly associated with patients having elevated troponin I levels. 
Table 1 Comparison of characteristic among troponin I and CPK-MB-positive and -negative individuals

\begin{tabular}{|c|c|c|c|c|c|c|c|}
\hline \multirow{2}{*}{\multicolumn{2}{|c|}{ Characteristic }} & \multicolumn{3}{|l|}{ Troponin I } & \multicolumn{3}{|l|}{ CPK-MB } \\
\hline & & Elevated & Normal & $p$-Value & Elevated & Normal & $p$-Value \\
\hline \multicolumn{2}{|c|}{ Age (mean \pm SD) } & $73.17 \pm 7.25$ & $58.84 \pm 13.66$ & 0.0176 & $70.45 \pm 6.876$ & $58.26 \pm 12.984$ & 0.0239 \\
\hline \multirow[t]{2}{*}{ Sex } & Male & 16 & 51 & 0.542 & 8 & 59 & 0.586 \\
\hline & Female & 12 & 21 & 0.694 & 6 & 27 & 0.763 \\
\hline \multicolumn{2}{|c|}{ Systolic blood pressure } & $182.6 \pm 26.5$ & $154.2 \pm 24.8$ & 0.254 & $174.98 \pm 38.46$ & $152.74 \pm 32.64$ & 0.476 \\
\hline \multicolumn{2}{|c|}{ Diastolic blood pressure } & $98.67 \pm 18.8$ & $90.3 \pm 19.7$ & 0.048 & $94.82 \pm 18.62$ & $93.58 \pm 19.38$ & 0.521 \\
\hline \multicolumn{2}{|c|}{ NIHS at admission } & $18-25$ & $6-16$ & 0.0126 & $12-25$ & $6-23$ & 0.0378 \\
\hline \multicolumn{2}{|c|}{ mRS at admission } & $4-5$ & $1-4$ & 0.0183 & $3-5$ & $1-5$ & 0.0467 \\
\hline \multicolumn{2}{|l|}{$\begin{array}{l}\text { Duration of } \\
\text { hospitalization }\end{array}$} & $12.116 \pm 3.158$ & $9.783 \pm 2.562$ & 0.0249 & $11.143 \pm 3.76$ & $9.254 \pm 2.178$ & 0.0285 \\
\hline \multicolumn{2}{|l|}{ Ischemic stroke } & 24 & 54 & 0.683 & 11 & 67 & 0.738 \\
\hline \multicolumn{2}{|c|}{ Hemorrhagic stroke } & 4 & 18 & 0.742 & 3 & 19 & 0.868 \\
\hline \multicolumn{2}{|l|}{ Mortality } & 3 & 3 & 0.564 & 4 & 2 & 0.0122 \\
\hline \multirow{2}{*}{$\begin{array}{l}\text { Left ventricular } \\
\text { hypertrophy } \\
\text { in Echo }\end{array}$} & + & 2 & 15 & \multirow[t]{2}{*}{0.869} & 0 & 17 & \multirow[t]{2}{*}{0.972} \\
\hline & - & 26 & 57 & & 14 & 69 & \\
\hline \multirow{2}{*}{$\begin{array}{l}\text { Regional } \\
\text { wall motion } \\
\text { abnormality } \\
\text { on Echo }\end{array}$} & + & 22 & 7 & \multirow[t]{2}{*}{0.0164} & 12 & 17 & \multirow[t]{2}{*}{0.0182} \\
\hline & - & 4 & 67 & & 2 & 69 & \\
\hline
\end{tabular}

Abbreviations: CPK-MB, creatine phosphokinase-MB; mRS, modified Rankin Score; NIHS, National Institute of Health Stroke; SD, standard deviation.

Table 2 Comparison of NIHS scores among patients with elevated and normal troponin T and CPK-MB

\begin{tabular}{|c|c|c|c|c|}
\hline \multirow[t]{2}{*}{ NIHS Score } & \multicolumn{2}{|c|}{ Troponin I } & \multicolumn{2}{|l|}{ СРК-МB } \\
\hline & Elevated & Normal & Elevated & Normal \\
\hline 0 & - & - & - & - \\
\hline $1-4$ & - & - & - & - \\
\hline $5-15$ & - & 66 & 1 & 65 \\
\hline $16-20$ & 14 & 6 & 5 & 15 \\
\hline $21-42$ & 14 & - & 8 & 6 \\
\hline$p$-Value & \multicolumn{2}{|l|}{$<0.0001$} & \multicolumn{2}{|l|}{0.2674} \\
\hline
\end{tabular}

Abbreviations: CPK-MB, creatine phosphokinase-MB; NIHS, National Institute of Health Stroke.

Table 3 Comparison of mRS scores among patients with elevated and normal CPK-MB

\begin{tabular}{|c|c|c|c|c|}
\hline & \multicolumn{2}{|c|}{ Troponin I } & \multicolumn{2}{|l|}{ CPK-MB } \\
\hline & Elevated & Normal & Elevated & Normal \\
\hline 0 & - & - & - & - \\
\hline 1 & - & 2 & - & 2 \\
\hline 2 & - & 39 & - & 39 \\
\hline 3 & - & 30 & 3 & 26 \\
\hline 4 & 19 & 1 & 5 & 10 \\
\hline 5 & 9 & - & 6 & 3 \\
\hline 6 & - & - & - & - \\
\hline$p$-Value & \multicolumn{2}{|l|}{$<0.0001$} & \multicolumn{2}{|l|}{0.0783} \\
\hline
\end{tabular}

Abbreviations: CPK-MB, creatine phosphokinase-MB; mRS, modified Rankin Score.

ECG changes were noted among $73 \%$ of study patients. $\mathrm{T}$ inversions were the commonest (54\%) finding followed by ST depression, ST elevation, and others such as sinus tachycardia and bradycardia, bundle branch block both right and left, multifocal atrial tachycardia, ventricular arrhythmia, and LVH. ST depression changes on the ECG were strongly associated with elevated troponin I and CPK-MB ( $p$-value 0.000). All the patients with elevated cardiac enzymes had ECG changes. 
Table 4 Mean NIHS and mRS scores among patients with elevated and normal troponin I and CPK-MB

\begin{tabular}{|c|c|c|c|c|c|c|}
\hline & & No. of patients & $\begin{array}{l}\text { NIHS } \\
\text { (mean) }\end{array}$ & $p$-Value & $\begin{array}{l}\text { mRS } \\
\text { (mean) }\end{array}$ & $p$-Value \\
\hline \multirow[t]{2}{*}{ Troponin I } & Normal & 72 & 10.5277 & \multirow[t]{2}{*}{$<0.001$} & 2.4305 & \multirow[t]{2}{*}{0.0034} \\
\hline & Elevated & 28 & 21.0357 & & 4.3214 & \\
\hline \multirow[t]{2}{*}{ CPK-MB } & Normal & 86 & 11.8721 & \multirow[t]{2}{*}{$<0.001$} & 2.756 & \multirow[t]{2}{*}{0.00467} \\
\hline & Elevated & 14 & 20.4285 & & 4.2143 & \\
\hline
\end{tabular}

Abbreviations: CPK-MB, creatine phosphokinase-MB; mRS, modified Rankin Score; NIHS, National Institute of Health Stroke.

Table 5 Comparison of the baseline characteristic among patients with elevated troponin I and CPK-MB

\begin{tabular}{|c|c|c|c|c|}
\hline & & Troponin I elevated & CPK-MB elevated & $p$-Value \\
\hline \multicolumn{2}{|c|}{ No. of patients } & 28 & 14 & 0.0116 \\
\hline \multicolumn{2}{|c|}{ Age (mean $\pm \mathrm{SD})$} & $73.17 \pm 7.25$ & $70.45 \pm 6.876$ & 0.0358 \\
\hline \multirow[t]{2}{*}{ Sex } & Male & 16 & 8 & \multirow[t]{2}{*}{0.0116} \\
\hline & Female & 12 & 6 & \\
\hline \multicolumn{2}{|c|}{ Systolic blood pressure } & $182.6 \pm 26.5$ & $174.98 \pm 38.46$ & 0.0413 \\
\hline \multicolumn{2}{|c|}{ Diastolic blood pressure } & $98.67 \pm 18.8$ & $94.82 \pm 18.62$ & 0.067 \\
\hline \multicolumn{2}{|c|}{ NIHS at admission } & $18-25$ & $12-25$ & 0.0238 \\
\hline \multicolumn{2}{|c|}{ mRS at admission } & $4-5$ & $3-5$ & 0.0572 \\
\hline \multicolumn{2}{|c|}{ Duration of hospitalization } & $12.116 \pm 3.158$ & $11.143 \pm 3.76$ & 0.0467 \\
\hline \multicolumn{2}{|c|}{ Ischemic stroke } & 24 & 11 & 0.0358 \\
\hline \multicolumn{2}{|c|}{ Hemorrhagic stroke } & 4 & 3 & 0.0794 \\
\hline \multicolumn{2}{|c|}{ Mortality } & 3 & 4 & 0.637 \\
\hline \multicolumn{2}{|c|}{ Left ventricular hypertrophy in Echo } & 2 & - & 0.001 \\
\hline \multicolumn{2}{|c|}{ Regional wall motion abnormality on Echo } & 22 & 12 & 0.0132 \\
\hline
\end{tabular}

Abbreviations: CPK-MB, creatine phosphokinase-MB; mRS, modified Rankin Score; NIHS, National Institute of Health Stroke; SD, standard deviation.

Seventeen patients had evidence of concentric LVH, 14 of them were known to have systemic hypertension, while three patients did not give any history of the same. Only three patients with elevated troponin I had LVH, while none with elevated CK-MB had LVH. Reduced ejection fraction (EF) was observed among 17 patients with elevated troponin I and 11 patients with elevated CK-MB. All the patients with reduced EF had evidence of regional wall motion abnormality on 2D echocardiography.

\section{Discussion}

Our study population had 67 men and 33 women participants with male-female ratio 2.03:1 that was similar to the studies by Nagaraja et al, Purushothaman et al, and Anand et al. ${ }^{9-11}$ Genetic susceptibility, estrogen effects on cerebral circulation, and lower blood pressure could all be the contributing factors for lower incidence of acute cerebrovascular accidents among the female population. Ischemic strokes were the commonest (78\%) among our study population and slightly more than previous studies by Kumar et al and Kuruvilla and Barucha et al. ${ }^{12,13}$

In a setting of acute stroke, the myocardial injury either due to ischemic or nonischemic etiology will be associated with elevated cardiac enzymes and ECG changes, with or without echocardiographic abnormalities. However, the nonischemic cardiac causes were ruled out, while including the patient in the study. Further carotid-vertebral Doppler was performed in all the study patients to substantiate the atherosclerotic etiology.

Several studies have studied the cardiac biomarkers in stroke patients. Most of these studies were on troponin $\mathrm{T}$ and CK-MB. We attempted at studying troponin I that is more cardiac specific and compared it with CK-MB. In a prospective study by Jensen et al, troponin $\mathrm{T}$ and $\mathrm{CPK}-\mathrm{MB}$ were found to be elevated among 10 and $9 \%$ of acute stroke patients, respectively, while $3 \%$ patients had both troponin T and CPK-MB elevated along with other ECG changes. ${ }^{14,15}$ In our study, 28\% patients had elevated troponin I, 14\% had elevated CPK-MB, 9\% had both troponin I and CPK-MB elevations and all the patients with elevated cardiac enzymes had ECG changes. These figures were slightly higher than those observed by Jensen et al, ${ }^{14,15}$ whereas in the study by Raza et al $8 \%$ patients had positive troponin I. ${ }^{16}$ Fure et al reported elevated troponin I among 9.6\% patients. ${ }^{17}$ Barber et al found $17.6 \%$ of the study population to have elevated troponin I and the elevation was associated with higher age and increased creatinine, ECG changes, and stroke severity. ${ }^{5}$

The increased levels of cardiac biomarkers in patients with acute ischemic stroke could be due to myocytolysis in cardiac muscles that would happen secondary to the activation of sympathetic nervous system, ${ }^{18}$ and probably increases 
the systemic catecholamines due to damage to the insular cortex. ${ }^{5}$ The B subunit of CK-MB could indicate brain damage that exists there as CK-BB which could also be an explanation of its elevation. ${ }^{8}$ Serum CK-MB was observed to be elevated in acute stroke patients in a Turkish study. ${ }^{19}$ However, we had only two patients with insular cortex involvement in our study.

Elevation of noradrenaline and adrenaline preceded elevation of CK-MB and troponin T in another study, which was associated with echocardiographic findings of new left ventricular wall motion abnormality and decreased cardiac output. High catecholamine levels in myocardium cause calcium overload in cardiac myocyte that impairs the cardiac contractility and cardiac function due to disturbance in capillary perfusion caused by increased platelet aggregation. ${ }^{20}$

In the index study, regional wall motion abnormalities on 2D echocardiogram were significantly associated with elevated troponin I but not associated with elevated CK-MB. All of these patients also had depressed EF. The findings were distinctly dissimilar to the study by Darki et al who noted that regional wall motion abnormality was significantly associated with elevated troponin I and CPK-MB levels. ${ }^{21}$ cTnI release was strongly associated with lower EF. ${ }^{22}$ Raza et $\mathrm{al}^{16}$ suggested the likely cause of low EF could be the post acute stroke surge in the sympathetic nervous system. The pathophysiology of depressed left ventricular EF in the setting of acute stroke is more likely due to sympathetic surge that leads to a transient decrease in the myocardial function. ${ }^{23}$

The underlying mechanisms in the myocardium mediating troponin I release differ from the mechanisms mediating CPK-MB release. The neurogenic influences could cause elevated CPK-MB without changes in troponin I. However, troponin I is found to be superior to CPK-MB in detecting early myocardial injury of both ischemic and nonischemic varieties. This makes it more likely that if a cardiac cause for CPK-MB elevation occurs then there would also be a concurrent elevation in troponin I levels. ${ }^{19,24,25}$

Anders et al proposed troponin I elevations without dynamic changes occur in stroke patients without acute coronary syndrome as a result of mechanisms stressing the heart. ${ }^{26}$ However, the magnitude of troponin elevation in patients with acute cerebrovascular accidents without evidence of coronary occlusion is less than that seen in coronary artery occlusion causing acute myocardial infarction. ${ }^{22,27,28}$ Cardiac troponin I is superior to CPK-MB in both its sensitivity and specificity in detecting minor myocardial injury. ${ }^{19}$

The presence of LVH is an independent risk factor for target organ damage due to systemic hypertension. ${ }^{29,30}$ Having clinical and echocardiographic evidence of LVH takes a longer duration, suggestive of probable pre-existing cardiac problems among some of these patients. The presence of LVH in patients with stroke doubles the risk of recurrent cerebrovascular accidents. ${ }^{31}$ Concentric LVH was observed among 17 patients in our study group. Only three patients with elevated troponin I had evidence of LVH but among none with elevated CK-MB.
The severity of stroke as predicted by higher NIHS and mRS scores was significantly associated with elevated troponin I and CPK-MB levels. This was in contrast to the observations made in some of the previous studies that showed severe strokes to be strongly correlated with elevated troponin I and not with CPK-MB. ${ }^{14,32}$ The mean NIHS scores were higher among patients with elevated troponin I but not among those with increased CPK-MB. This implies that patients with increased troponin I had more severe forms of strokes than those with normal troponin I values. But CPK-MB could not show such correlation.,32-34

Elevated troponin I was a better predictor of morbidity (duration of hospital stay) than elevated CK-MB in our study. Significant changes in mortality with elevated troponin I were not observed in our study, which was in strong disagreement with the observations of James et al, who concluded elevated troponin on admission in patients with acute stroke as a strong predictor of mortality and poor prognosis ${ }^{35}$; Fure et al, who demonstrated poor outcomes over short-term among patients with elevated troponin $\mathrm{T}^{17}$ and Raza et al, who concluded elevated troponins predict long-term cardiac outcomes among patients with acute stroke with no evidence of cute coronary event. ${ }^{16}$

The study results raise the debate whether routine checking of cardiac enzymes in acute stroke patients should be performed or not. Etgen et al did serial measurements of cTnT and cTnI at admission on day 1 and day 2 . They observed that $7.8 \%$ had highest troponin level on day 2 and $4.6 \%$ had highest level on day 3; thus, they recommended that routine measurement of troponin had no significant contribution in the evaluation of acute stroke patients. ${ }^{7}$ The American Stroke Association $^{36}$ does recommend customary measurement of the cardiac biomarkers, while the Scottish Intercollegiate Guidelines Network ${ }^{37}$ and the National Institute of Clinical excellence-based UK acute stroke guidelines ${ }^{38}$ do not recommend this. Cardiac troponin levels may be useful among patients with acute stroke who may need early coronary artery disease evaluation for secondary prevention. ${ }^{27}$

\section{Limitations}

We did not include healthy control group, which could have helped us comparing the mean values of troponin I and CPK-MB among acute stroke patients and the controls. The sympathetic nervous activity was not studied as it was beyond the scope of the present study.

\section{Conclusion}

Higher levels of cardiac enzymes in patients of acute cerebrovascular accidents without previous history of cardiac disease are significantly correlated with the severity of stroke. These patients need to be considered for early coronary evaluation post stroke. However, routine screening of these cardiac enzymes in the patients with stroke is not required but can be considered when there are ECG changes or evidence of reduced EF with or without regional wall motion abnormalities on echocardiogram. 


\section{Conflict of Interest}

None declared.

\section{References}

1 Katsiki N, Ntaios G, Vemmos K. Stroke, obesity and gender: a review of the literature. Maturitas 2011;69(3):239-243

2 Lynn S. Cardiovascular news, The American Heart Association. Circulation 2000;102:502-504

3 Song HS, Back JH, Jin DK, et al. Cardiac troponin T elevation after stroke: relationships between elevated serum troponin $\mathrm{T}$, stroke location, and prognosis. J Clin Neurol 2008;4(2):75-83

4 Levy A. The exiting causes of ventricular fibrillation in animals under chloroform anaesthesia. Heart 1993;4:319-378

5 Barber M, Morton JJ, Macfarlane PW, Barlow N, Roditi G, Stott DJ. Elevated troponin levels are associated with sympathoadrenal activation in acute ischaemic stroke. Cerebrovasc Dis 2007;23(4):260-266

6 WHO MONICA Project Principal Investigators. The World Health Organization MONICA Project (monitoring trends and determinants in cardiovascular disease): a major international collaboration. J Clin Epidemiol 1988;41(2):105-114

7 Etgen T, Baum H, Sander K, Sander D. Cardiac troponins and $\mathrm{N}$-terminal pro-brain natriuretic peptide in acute ischemic stroke do not relate to clinical prognosis. Stroke 2005;36(2):270-275

8 Bell RD, Alexander GM, Nguyen T, Albin MS. Quantification of cerebral infarct size by creatine kinase BB isoenzyme. Stroke 1986;17(2):254-260

9 Nagaraja D, Gururaj G, Girish N, et al. Feasibility study of stroke surveillance: data from Bangalore, India. Indian J Med Res 2009;130(4):396-403

10 Purushothaman S, Salmani D, Prarthana KG, Bandelkar SMG, Varghese S. Study of ECG changes and its relation to mortality in cases of cerebrovascular accidents. J Nat Sci Biol Med 2014;5(2):434-436

11 Anand K, Chowdhury D, Singh KB, Pandav CS, Kapoor SK. Estimation of mortality and morbidity due to strokes in India. Neuroepidemiology 2001;20(3):208-211

12 Kumar HH, Kalra B, Goyal N. A study on stroke and its outcome in young adults (15-45 years) from coastal South India. Indian J Community Med 2011;36(1):62-65

13 Kuruvilla T, Bharucha NE. Epidemiology of stroke in India. Neurol J Southeast Asia. 1998;3:5-8

14 Jensen JK, Atar D, Mickley H. Mechanism of troponin elevations in patients with acute ischemic stroke. Am J Cardiol 2007; 99(6):867-870

15 Jensen JK, Kristensen SR, Bak S, Atar D, Høilund-Carlsen PF, Mickley H. Frequency and significance of troponin T elevation in acute ischemic stroke. Am J Cardiol 2007;99(1):108-112

16 Raza F, Alkhouli M, Sandhu P, Bhatt R, Bove AA. Elevated cardiac troponin in acute stroke without acute coronary syndrome predicts long-term adverse cardiovascular outcomes. Stroke Res Treat 2014;2014:621650

17 Fure B, Bruun Wyller T, Thommessen B. Electrocardiographic and troponin $\mathrm{T}$ changes in acute ischaemic stroke. J Intern Med 2006;259(6):592-597

18 Myers MG, Norris JW, Hachinski VC, Weingert ME, Sole MJ. Cardiac sequelae of acute stroke. Stroke 1982;13(6):838-842

19 Ay H, Arsava EM, Saribaş O. Creatine kinase-MB elevation after stroke is not cardiac in origin: comparison with troponin T levels. Stroke 2002;33(1):286-289

20 Batal O, Jentzer J, Balaney B, et al. The prognostic significance of troponin I elevation in acute ischemic stroke. J Crit Care 2016;31(1):41-47

21 Darki A, Schneck MJ, Agrawal A, Rupani A, Barron JT. Correlation of elevated troponin and echocardiography in acute ischemic stroke. J Stroke Cerebrovasc Dis 2013;22(7):959-961
22 Tung P, Kopelnik A, Banki N, et al. Predictors of neurocardiogenic injury after subarachnoid hemorrhage. Stroke 2004; 35(2):548-551

23 Francis GS, Goldsmith SR, Levine TB, Olivari MT, Cohn JN. The neurohumoral axis in congestive heart failure. Ann Intern Med 1984;101(3):370-377

24 Rottbauer W, Greten T, Müller-Bardorff M, et al. Troponin T: a diagnostic marker for myocardial infarction and minor cardiac cell damage. Eur Heart J 1996;17(Suppl F) :3-8

25 Lang K, Börner A, Figulla HR. Comparison of biochemical markers for the detection of minimal myocardial injury: superior sensitivity of cardiac troponin-T ELISA.J Intern Med 2000; 247(1):119-123

26 Anders B, Alonso A, Artemis D, et al. What does elevated high-sensitive troponin I in stroke patients mean: concomitant acute myocardial infarction or a marker for high-risk patients? Cerebrovasc Dis 2013;36(3):211-217

27 Mochmann HC, Scheitz JF, Petzold GC, et al; TRELAS Study Group. Coronary angiographic findings in acute ischaemic stroke patients with elevated cardiac troponin: the Troponin Elevation in Acute Ischaemic Stroke (TRELAS)study. Circulation 2016;133(13):1264-1271

28 Homma S, Grahame-Clarke C. Editorial comment-myocardial damagein patientswithsubarachnoidhemorrhage.Stroke2004 35(2):552-553

29 Bikkina M, Levy D, Evans JC, et al. Left ventricular mass and risk of stroke in an elderly cohort. The Framingham Heart Study. JAMA 1994;272(1):33-36

30 Kannel WB. Prevalence and natural history of electrocardiographic left ventricular hypertrophy. Am J Med 1983; 75(3A) :4-11

31 Pop GA, Koudstaal PJ, Meeder HJ, Algra A, van Latum JC, van Gijn J; The Dutch TIA Trial Study Group. Predictive value of clinical history and electrocardiogram in patients with transient ischemic attack or minor ischemic stroke for subsequent cardiac and cerebral ischemic events. Arch Neurol 1994; 51(4):333-341

32 Scheitz JF, Endres M, Mochmann HC, Audebert HJ, Nolte $\mathrm{CH}$. Frequency, determinants and outcome of elevated troponin in acute ischemic stroke patients. Int J Cardiol 2012; 157(2):239-242

33 Král M, Šaňák D, Veverka T, et al. Troponin T in acute ischemic stroke. Am J Cardiol 2013;112(1):117-121

34 HaiderAh,Al-KhefajiDK,AbbaseAh,etal.Prognosticsignificance of troponin $\mathrm{T}$ and creatine kinase-MB activity in sera of acute ischaemic stroke patients. Medical Journal of Babylon 2010;7: 469-479

35 James P, Ellis CJ, Whitlock RM, McNeil AR, Henley J, Anderson NE. Relation between troponin T concentration and mortality in patients presenting with an acute stroke: observational study. BMJ 2000;320(7248) :1502-1504

36 American Heart Association/ American Stroke Association Guideline. Guidelines for the Early Management of Adults with Ischaemic Stroke. Dallas: AHA; 2007. Available from: http://www.stroke.ahajournals.org/cgi/reprint/STROKEAHA. 107.181486. Accessed Jul 17, 2017

37 Scottish Intercollegiate Guidelines Network. Management of Patients with Stroke: Assessment, Investigation, Immediate Management and Secondary Prevention (draft). Glasgow: SIGN; 2008

38 National Institute of Clinical Excellence: Acute Stroke and TIA Clinical Guidelines (draft). London: National Institute of Clinical Excellence; 2008. Available at: http://www.nice. org.uk/guidance/index.jsp?action=byID\&o=11646. Accessed Jul 17, 2017 